\title{
ON THE RANGE OF FINITE EMBEDDINGS OF A FINITE AMALGAM
}

\author{
by ABDUL MAJEED
}

(Received 12 October, 1970)

Certain questions about the range of finite embeddings of a finite amalgam were discussed in [3]. Another pertinent question is the following.

If a finite reduced amalgam has both an infinite and a finite embedding, does it have a maximal finite embedding such that all other finite embeddings are its homomorphic images?

We give a counter example to answer this question in the negative. The finite amalgam considered will involve a group from the family of groups of the type $(l, m ; n, k)$ discussed by Coxeter [2] having the following presentation:

$$
(l, m ; n, k)=\mathrm{gp}\left\{g, h ; g^{l}=h^{m}=(g h)^{n}=\left(g^{-1} h\right)^{k}=1\right\} .
$$

These groups may be regarded as factor groups of

$$
\operatorname{gp}\left\{g, h ; g^{l}=h^{m}=(g h)^{n}=1\right\},
$$

which is known to be finite if

$$
\frac{1}{l}+\frac{1}{m}+\frac{1}{n}>1
$$

and infinite otherwise.

When $l=m=n=3$, then (2) becomes

$$
G_{3}=\operatorname{gp}\left\{g, h ; g^{3}=h^{3}=(g h)^{3}=1\right\}
$$

and is therefore infinite. It is shown in [1] that every element of $G_{3}$ is expressible in the form

$$
h^{r}\left(g h^{-1}\right)^{p}\left(g^{-1} h\right)^{q} \quad(p, q, r \text { integers }) .
$$

Therefore the most general factor group of $G_{3}$ is given by

$$
\left(g h^{-1}\right)^{p}\left(g^{-1} h\right)^{q}=1,
$$

which takes the form $\left(g h^{-1} g^{-1} h\right)^{q}=1$ when $p=q$. The relation $\left(g h^{-1} g^{-1} h\right)^{q}=1$ implies $\left(g^{-1} h\right)^{3 q}=1$. The group

$$
(3,3 ; 3, k)=\mathrm{gp}\left\{g, h ; g^{3}=h^{3}=(g h)^{3}=\left(g^{-1} h\right)^{k}=1\right\}
$$

is finite of order $3 k^{2}$ for every $k \geqq 2$ and is a central quotient group of the group $H_{3}$ given by

$$
H_{3}=\operatorname{gp}\left\{g, h ; g^{3}=h^{3}=(g h)^{3}=\left(g h^{-1} g^{-1} h\right)^{k}=1\right\} .
$$

The relation (4) shows that there is no minimal normal subgroup of $G_{3}$. 
Consider now the finite amalgam $\mathbf{A}$ formed by the groups

$$
\begin{aligned}
& A=\operatorname{gp}\left\{a, b ; a^{2}=b^{2}=(a b)^{3}=1\right\}, \\
& B=\operatorname{gp}\left\{b, c ; b^{2}=c^{2}=(b c)^{3}=1\right\}, \\
& C=\operatorname{gp}\left\{c, a ; c^{2}=a^{2}=(c a)^{3}=1\right\} .
\end{aligned}
$$

The free embedding of $\mathrm{A}$ is

$$
F=\operatorname{gp}\left\{a, b, c ; a^{2}=b^{2}=c^{2}=(a b)^{3}=(b c)^{3}=(c a)^{3}=1\right\} .
$$

Take $b c=g, c a=h$; then $b a=g h$. Then $F$ has an alternative presentation:

$$
F=\operatorname{gp}\left\{g, h, c ; g^{3}=h^{3}=(g h)^{3}=(g c)^{2}=(c h)^{2}=c^{2}=1\right\} .
$$

Since

$$
c^{-1} g c=c \cdot b c \cdot c=c b=(b c)^{-1}=g^{-1}
$$

and

$$
c^{-1} h c=c \cdot c a \cdot c=a c(c a)^{-1}=h^{-1},
$$

$F$ is a split extension of $G_{3}$ by a cycle of order 2. Therefore $F$ is an infinite embedding of A.

Also, for each integer $k$, the normal closure $N_{k}$ of $\left(g^{-1} h\right)^{k}$ in $G_{3}$ is normal in $F$. The relation $\left(g^{-1} h\right)^{k}=1$ implies $(c b c a)^{k}=1$. For $k>2, N_{k}$ is tidy with respect to $A, B, C . F / N_{k}$, therefore, embeds the amalgam $A$. But

$$
F / N_{k}=\operatorname{gp}\left\{g, h, c ; g^{3}=h^{3}=(g h)^{3}=\left(g^{-1} h\right)^{k}=(g c)^{2}=(c h)^{2}=c^{2}=1\right\},
$$

being an extension of $(3,3 ; 3, k)$ of order $3 k^{2}$ by a cycle of order 2 , is finite. Thus $A$ has a finite embedding, namely $F / N_{k}$. Since the element $c$ normalises $G_{3}$, all the finite embeddings of A are determined by the normal subgroups of $G_{3}$. As shown above, since $G_{3}$ has no minimal normal subgroup, $A$ has no maximal finite embedding having each of the finite embeddings $F / N_{k}$ as its homomorphic images.

The author is grateful to the referee of [3] for suggesting this problem.

\section{REFERENCES}

1. W. Burnside, Theory of groups of finite order (Cambridge, 1911).

2. H. S. M. Coxeter, The abstract groups $G^{m, n, p}$, Trans Amer. Math. Soc. 45 (1939), 73-150.

3. A. Majeed, On embeddable finite amalgams of groups, Glasgow Math. J. 13 (1972), 135-141.

UNIVERSITy OF THE PUNJAB

LAHORe, Pakistan 